\title{
Physical Medicine and Rehabilitation Research
}

\section{Quantitative assessment of Muscle stiffness using Tensiomyography before and after Injection of Botulinum toxin Type A in Patients after Stroke}

\author{
Yukio Mikami ${ }^{1,2 *}$, Kai Ushio ${ }^{2,3}$, Akihiro Matsumoto ${ }^{2}$, Ken Kouda ${ }^{1}$, Hiroaki Kimura ${ }^{2,3}$ and Nobuo Adachi ${ }^{3,4}$ \\ ${ }^{1}$ Department of Rehabilitation Medicine, Wakayama Medical University, Japan \\ ${ }^{2}$ Department of Rehabilitation Medicine, Hiroshima University Hospital, Japan \\ ${ }^{3}$ Sports Medical Center, Hiroshima University Hospital, Japan \\ ${ }^{4}$ Department of Orthopedic Surgery, Graduate School of Biomedical Sciences, Hiroshima University, Japan
}

\begin{abstract}
Background: There have been still few objective outcome measures regarding the effect of botulinum toxin type A treatment. Tensiomyography (TMG) is used as a device that can non-invasively evaluate skeletal muscle, however, no reports have been found that the effects of botulinum toxin type A treatment have been evaluated by the characteristics of muscle contraction using TMG.
\end{abstract}

Objective: To evaluate the clinical usefulness of quantitative assessment using TMG before and after injection of botulinum toxin type A in patients after stroke.

Methods: The subjects were 10 patients who received botulinum toxin type A treatment for the spasticity of biceps brachii muscle after stroke. The maximum displacement $(\mathrm{Dm})$ values in TMG of the affected side and the unaffected side before botulinum toxin type A administration were compared. Then, changes in Dm values, Modified Ashworth Scale (MAS) of elbow joint and Visual Analogue Scale (VAS) on the affected side were examined. In addition, the correlation between the MAS values and the Dm values, between the VAS values and the Dm values were examined.

Results: The Dm values before botulinum toxin type A injection was significantly lower on the affected side than that on the unaffected side. The Dm values and the VAS values one month after administration were significantly higher and lower than those before administration and three months after administration, respectively. Although there was a low correlation between the MAS value and the Dm value, a moderate correlation was observed between the VAS value and the Dm value.

Conclusion: There are few objective outcome measures regarding the therapeutic effect of botulinum toxin therapy. However, measurement of the characteristics of muscle contraction using TMG might be useful as one of the quantitative outcome measures for the effect of botulinum toxin type A therapy in patients after stroke.

Abbreviations: TMG: Tensiomyography; Dm: Maximum Displacement; MAS: Modified Ashworth Scale; VAS: Visual Analogue Scale; BT: Botulinum Toxin Type A; ANOVA: Analysis of Variance; SD: Standard Deviation; ARFI: Acoustic Radiation Force Impulse.

\section{Introduction}

Muscle spasticity after stroke causes pain and decrease of activities of daily living (ADL), and is one of the causes of decreasing Quality of life (QOL) and social participation of the patients. Botulinum toxin is a neurotoxin produced by Clostridium botulinum, and type A is the most stable and toxic [1]. In 1977, Scott [2] first applied botulinum toxin type A (BTA) clinically for strabismus, and was also used for the treatment of blepharospasm, hemifacial spasm, and spastic torticollis. In recent years, BTA has become widely applied as a treatment for muscle spasticity after stroke, and there have been many reports that said BTA treatment is a safe and effective treatment [3-5]. BTA cleaves the SNAP25 protein involved in the release of acetylcholine within nerve endings at the neuromuscular junction. Thereby, the release of acetylcholine is suppressed, and the neuromuscular transmission is suppressed to obtain muscular relaxation. Neurons in which neuromuscular transmission has been inhibited are reopened several months later due to the formation of a nerve branch from the axonal side, and the muscular relaxation disappears [6]. The effect of BTA treatment on muscle spasticity is generally assessed using the modified Ashworth scale (MAS). However, the evaluation by MAS is less objective and has differences among the examiners, and MAS is not highly reliable [7-9]. Therefore, there have been few objective outcome measures regarding the effect of BTA treatment, and the optimal dose, duration of the effect, and the interval of re-administration of BTA have not been clarified.

Recently, tensiomyography (TMG) is used as a device that can non-invasively evaluate skeletal muscle, particularly in the sports field [10-12]. TMG applies electrical stimulation to the skin surface, and it is possible to evaluate the characteristics of muscle contraction such as maximum displacement $(\mathrm{Dm})$ from the change in shape of the

${ }^{*}$ Correspondence to: Yukio Mikami, MD, Ph.D., Department of Rehabilitation Medicine, Wakayama Medical University, Japan, E-mail: ymikami@wakayamamed.ac.jp

Key words: tensiomyography, stroke, spasticity, botulinum toxin type A, quantitative assessment

Received: October 25, 2019; Accepted: October 30, 2019; Published: November 07,2019 
center of the muscle. The measurements using TMG is reported to be highly reliable [13-15], and Rey et al. also reported that the Dm in TMG is related to muscle stiffness [16]. TMG is non-invasive and can be used to evaluate muscle contraction quickly, so it is often used in the fields of sports and rehabilitation medicine, and there have been some reports about sports injuries and trainings [17-20].

However, so far, no reports have been found that the effects of BTA treatment have been evaluated by the characteristics of muscle contraction using TMG. We have clinically measured the characteristics of muscle contraction in patients after stroke and observed their changes after BTA injection using TMG. Therefore, we aimed to evaluate the clinical usefulness of quantitative assessment using TMG before and after injection of BTA in patients after stroke. This study is the first report to evaluate the muscle stiffness and the effects of BTA therapy in patients after stroke using TMG.

Our hypothesis is that in the patients after stroke, Dm values in TMG decrease along with increase of MAS and VAS values because of increased muscle stiffness, and after administration of BTA, MAS and VAS values decrease and Dm values increase for a certain period. In addition, we hypothesized that there is correlation between MAS and Dm values and between VAS and Dm values.

\section{Subjects and methods}

\section{Subjects}

Medical records were retrospectively surveyed of 31 people who received BTA treatment for the muscle spasticity after stroke at the Department of Rehabilitation Medicine in Hiroshima University Hospital and related hospitals. Inclusion criteria include, 1) those who received BTA treatment for spasticity of biceps brachii muscle after stroke, 2) those who were measured the characteristics of muscle contraction of biceps brachii muscle using TMG as well as MAS in the elbow joint and VAS of the biceps brachii muscle before and after BTA injection, 3) those who had the muscle spasticity more than MAS 1 in the elbow joints on the affected side ,4) those who had the pain in biceps brachii muscle because of the muscle spasticity. Exclusion criteria include, 1) those who had difficulty in communication because of severe higher brain dysfunction, 2) those who had surgery or other treatment for muscle spasticity, 3) those who could not be obtained informed consent for the research.

16 patients out of 31 patients met inclusion criteria, 6 of which were excluded, and finally 10 patients ( 6 male, 4 female, average age $60.6 \pm$ 10.5 years) were included in the study.

\section{Methods}

TMG-100 ${ }^{\mathrm{TM}}$ (TMG-BMC Ltd., Ljubljana, Slovenia) was used as a measurement device for TMG. Before administration of BTA, Dm value of biceps brachii muscle on the affected side as well as the unaffected side was measured using TMG. According to the method of Rey et al. [16], the measurement method was as follows, a) The sensor rod was placed at the center of the biceps brachii muscle, and the electrode was set so as to sandwich the rod, b) In a relaxed state, the tip of the sensor rod was inserted to half of the rod, c) The stimulation was started from $20 \mathrm{~mA}, \mathrm{~d}$ ) The stimulation was gradually increased until the change in Dm disappeared, or up to a maximum of $60 \mathrm{~mA}$, and the maximum value was taken as the Dm value (Figure 1). In addition, spasticity of the affected elbow joint was evaluated by MAS, and pain in the biceps brachii muscle was evaluated by Visual Analogue Scale
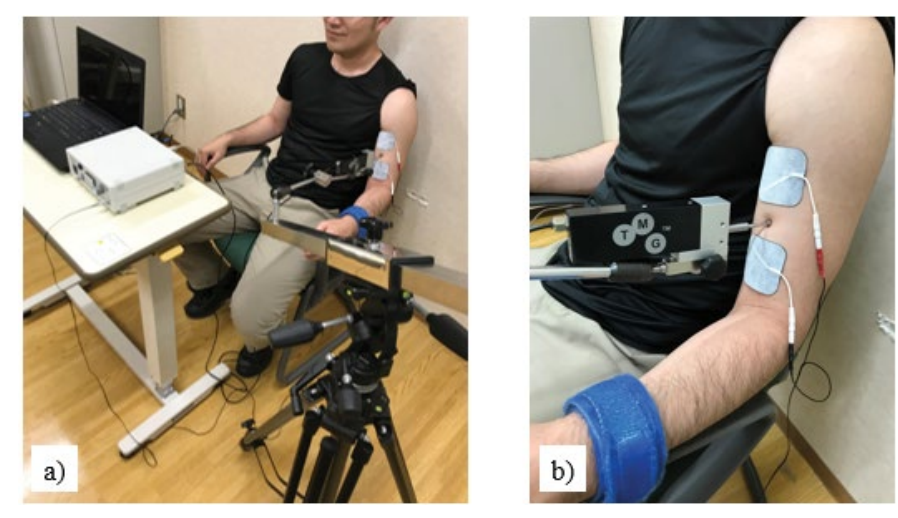

Figure 1. Measurement of the characteristics of muscle contraction using tensiomyography (TMG)

a. TMG-100 (TMG-BMC Ltd., Ljubljana, Slovenia) was used as a device for TMG. TMG applies electrical stimulation to the skin surface, and it is possible to evaluate the characteristics of muscle contraction.

b. The sensor rod was placed at the center of the biceps brachii muscle, and the electrode was set so as to sandwich the rod. In a relaxed state, the tip of the sensor rod was inserted to half of the rod.

(VAS). For statistical analysis of MAS, 1+ was considered as 2, 2 as 3,3 as 4 , and 4 as 5 according to the report of Ceyhan Bilgici $\mathrm{M}$ et al. [21]. Regarding VAS, the patients were shown a $10 \mathrm{~cm}$ long black line (left side is without pain and right side is with extreme pain that could be imagined) and were asked to indicate how much the pain was. The dosage of BTA (Botox, Allergan, Inc., CA, USA) was determined according to the spasticity of each patient and was administered to the center of muscle belly in biceps brachii on the affected side. At 1 month and 3 months after administration, the Dm value of the biceps brachii muscle on the affected side was measured again using TMG, and the MAS value of the elbow joint and the VAS value of the biceps brachii muscle were also measured. Administration of BTA was performed by one physician and measurement of TMG was performed by one skilled physician for a total of three times before and after administration of BTA in one patient.

Diagnosis of the subject, period after onset, administration history of BTA, administration method, post-administration rehabilitation, side effects, measurement results of Dm values of biceps brachii muscle, MAS values of the affected elbow joint, VAS values of the affected brachial biceps muscle were examined retrospectively from medical records. The Dm values of the affected side and the unaffected side before the administration of BTA were compared. Then, changes in Dm value of biceps brachii muscle, MAS value of elbow joint and VAS value of biceps brachii muscle on the affected side were examined. In addition, the correlation between MAS values of elbow joint and Dm values of biceps brachii muscle, between VAS values and Dm values of biceps brachii muscle on the affected side before and after administration of BTA were examined.

Statistical analysis: Continuous variables were expressed as the mean \pm standard deviation (SD). In comparison of $\mathrm{Dm}$ values between affected and unaffected side of biceps brachii muscle before administration of BTA, a paired t-test was used, with $\mathrm{p}<0.05$ regarded as significant. The measured values of Dm of biceps brachii muscle, MAS of elbow joint, VAS of biceps brachii muscle on the affected side before administration of BTA and at one month and three months after administration were confirmed by the Shapiro-Wilk test according to whether each variable followed the normal distribution. Then, when the main effect was significant in the repeated measures analysis of 
variance (ANOVA), the Tukey HSD method was used as a post-hoc test. Furthermore, in order to examine the correlation between the affected side of MAS value of elbow joint and Dm value of biceps brachii muscle, between the affected side of VAS value and Dm value of biceps brachii muscle, Pearson's correlation coefficients were determined.

Data analysis was performed using JMP ${ }^{\circ}$ pro version 14 (SAS Institute Inc.) and $\mathrm{p}<0.05$ was regarded as significant in all outcome measures.

This study was approved by the Research Ethics Review Committee of the Hiroshima University Hospital (No. E-1483).

\section{Results}

Of the ten patients, five were post-cerebral hemorrhage, four were post-cerebral infarction and one was post-subarachnoid hemorrhage. The average time from onset to measurement was $80.1 \pm 43.3$ months. Two of the ten patients were patients on the first injection, while eight were patients on ongoing injections. The doses of BTA to the biceps brachii muscle were all 50 units, and all were injected in one place. After administration, four had regular outpatient rehabilitation by a physical therapist twice a week, and one had home visiting rehabilitation by a physical therapist once a week, however, five performed only selfexercise at home. All ten patients had no apparent side effects after administration, and TMG measurements were completed.

The example of the results in a case are illustrated in (Figure 2). In that case, the Dm value of biceps brachii muscle before BTA administration was $10.4 \mathrm{~mm}$ on the unaffected side and $2.6 \mathrm{~mm}$ on the affected side. At one month after administration, the Dm value of the affected side increased to $6.1 \mathrm{~mm}$, and at three months after administration, it decreased to $3.6 \mathrm{~mm}$.

The mean Dm value $( \pm \mathrm{SD}$ ) of the biceps brachii muscle before administration of BTA in ten subjects is $8.8 \pm 2.6 \mathrm{~mm}$ on the unaffected side and $3.2 \pm 0.9 \mathrm{~mm}$ on the affected side, and the Dm value on the affected side was significantly lower than that on the unaffected side $(p<0.001)$. Table 1 shows the changes in Dm, MAS, and VAS values before and after BTA treatment on the affected side. The mean Dm value ( \pm SD) of biceps brachii muscle on the affected side increased to $5.2 \pm$ $1.3 \mathrm{~mm}$ at first month and decreased to $3.6 \pm 1.0 \mathrm{~mm}$ at 3 months after administration. The mean VAS value $( \pm S D)$ of the biceps brachii muscle on the affected side was $51.8 \pm 7.6$ before administration, decreased to $34.9 \pm 5.5$ at first month, and increased to $42.9 \pm 6.4$ at three months after administration. The Dm values $( \pm S D)$ and the VAS values $( \pm S D)$ of the biceps brachii muscle on the affected side showed significant differences in the repeated-measures analysis of variance (ANOVA). In the post-hoc test, the Dm value and the VAS values one month after administration were significantly higher and lower than those before administration and three months after administration, respectively. The mean MAS value $( \pm$ SD) of the affected elbow joint was $3.1 \pm 0.5$ before administration, decreased to $2.6 \pm 0.5$ at first month, and increased to $2.9 \pm 0.3$ at three months after administration. The MAS values $( \pm$ SD) of the affected elbow joint did not show significant differences in the repeated-measures ANOVA.

Although there was a low correlation between the MAS value of the elbow joint and the Dm value of biceps brachii muscle on the affected side before and after administration of BTA $\left(R^{2}=-0.237\right.$, $\mathrm{P}=0.006)$, a moderate correlation was observed between the VAS value and the $D m$ value of biceps brachii muscle $\left(R^{2}=-0.646, P<0.001\right)$ (Figure 3).

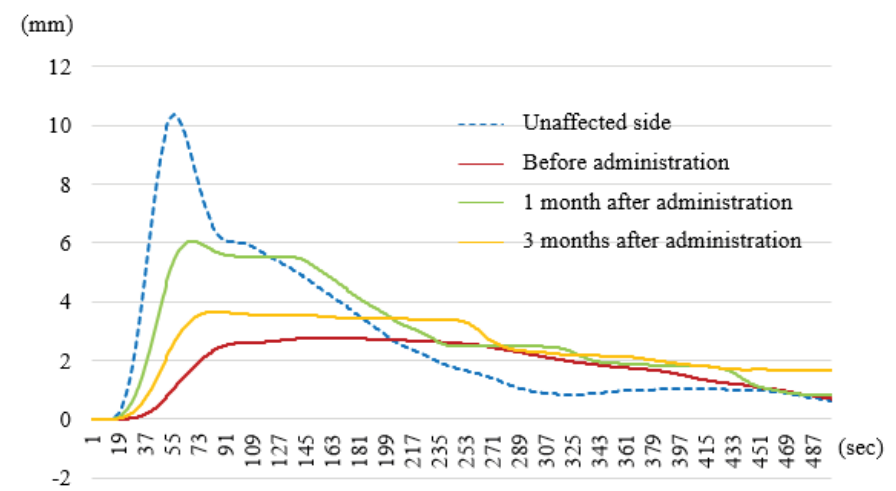

Figure 2. The measured $\mathrm{Dm}$ values in a case. The $\mathrm{Dm}$ value of biceps brachii muscle before botulinum toxin type A administration was $10.4 \mathrm{~mm}$ on the unaffected side and 2.6 $\mathrm{mm}$ on the affected side. At one month after administration, the Dm value of the affected side increased to $6.1 \mathrm{~mm}$, and at three months after administration, it decreased to $3.6 \mathrm{~mm}$

$(\mathrm{mm})$

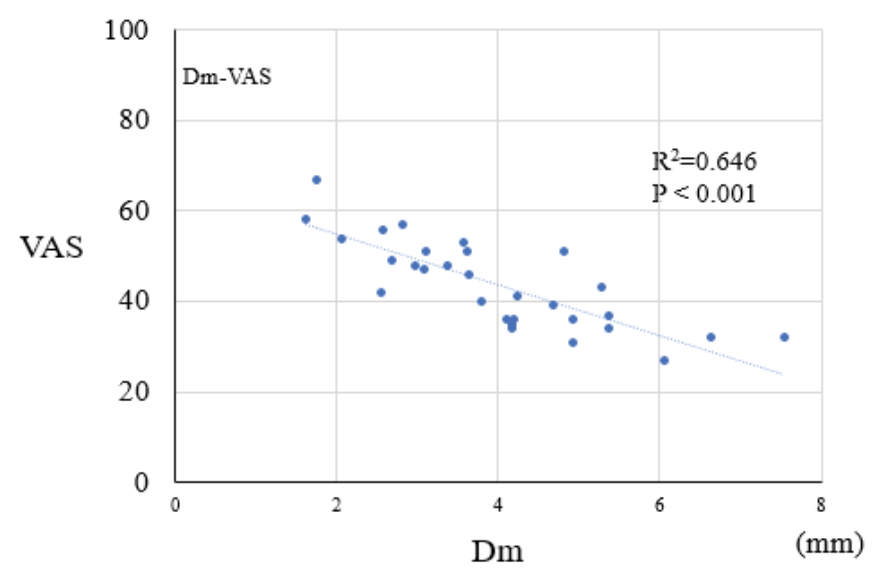

Figure 3. The correlation between VAS values and Dm values of biceps brachii muscle on the affected side before and after administration of botulinum toxin type A. Pearson's correlation coefficients were determined

Table 1. Changes in Dm, MAS, and VAS before and after BTA treatment

\begin{tabular}{|c|c|c|c|}
\hline & $\begin{array}{c}\text { Before BTA } \\
\text { injection }\end{array}$ & $\begin{array}{c}\text { 1M after BTA } \\
\text { injection }\end{array}$ & $\begin{array}{c}\text { 3M after BTA } \\
\text { injection }\end{array}$ \\
\hline Dm & $3.1 \pm 0.9$ & $5.2 \pm 1.3^{*} \dagger$ & $3.6 \pm 1.0$ \\
\hline MAS & $3.1 \pm 0.5$ & $2.6 \pm 0.5$ & $2.9 \pm 0.3$ \\
\hline VAS & $51.8 \pm 7.6$ & $34.9 \pm 5.5^{*} \dagger$ & $42.9 \pm 6.4$ \\
\hline
\end{tabular}

BTA: botulinum toxin type A; Dm: maximum displacement; MAS: modified Ashworth scale; VAS: Visual Analogue Scale. ${ }^{*}, \mathrm{p}<0.05$ vs Before BTA injection $\uparrow, \mathrm{P}<0.05$ vs $3 \mathrm{M}$ after BTA injection

\section{Discussion}

This study is the first report of measuring the characteristics of muscle contraction using TMG for the patients after stroke, and the changes of muscle contraction characteristics after BTA administration was also evaluated using TMG for the first time.

Since Dm values in TMG have been reported to be related to muscle stiffness [16], it was quantitatively shown that the muscle stiffness of the affected biceps brachii muscle is higher than that in the unaffected side using TMG. In addition, at one month after BTA administration, muscle stiffness of the affected biceps brachii muscle decreased significantly, and the effect of BTA administration was clearly observed, however, no significant decrease in muscle stiffness 
was observed at three months after administration, and the effect of BTA was reduced at three months after administration. The effect of BTA is reversible, and so far, the scientific basis for the interval of re-administration has not been clear. However, since the results of this study quantitatively show that the effect of BTA is diminished at 3 months, re-administration at 3 months after administration could be accepted. The MAS value in the affected elbow joint tended to decrease at one month after administration, however that was not significant. In addition to the biceps brachii muscle, the flexion of the elbow joint involves the brachial muscle and the brachioradial muscle. However, in this study, BTA was not administered to these muscles in all subjects and the administration of BTA to the biceps brachii muscle was at one place and 50 units. Thus, the site and dose of BTA in this study might not have been sufficient to appreciably improve spasticity in the affected elbow joint. Or, because MAS itself is not highly objective and reliable, the slight improvement in spasticity obtained in this study might not be able to evaluate. However, in this study, the change corresponding to the Dm value of the biceps brachii muscle was observed in the VAS value and administration of BTA in this study is effective in the affected biceps brachii muscle and the patients' complaints in biceps brachii muscle have been improved at one month after administration. Currently, since there is no clear guideline on the administration of BTA, the administration dose and site of BTA, and the selection of administration muscle are appropriately changed in consideration of patient complaints and ADL. Thus, in the future, it is necessary to adjust the dosage and location of BTA and the muscle to be administered based on quantitative functional evaluation as well as evaluation on activity, participation and QOL.

In an attempt to quantify muscle stiffness, a method has been used in which the displacement of the entire subcutaneous tissue when pressure is applied from the surface of a body is quantified using a "press-in type muscle hardness meter" [22]. However, since the reaction force of the tissue obtained by this method also includes the "hardness" of the skin and subcutaneous fat, it cannot be said that it directly reflects the "stiffness" of the muscle itself. In addition, because the measurement by the muscle hardness meter involves the measurement error by the examiner, its validity and reliability are not so high. On the other hand, as described above, TMG is reported to be non-invasive and highly reliable and reproducible [13-15].

The systematic review by Mathevon L, et al. reported that ultrasound elastography was non-invasive and useful in the evaluation of muscle stiffness after administration of BTA [23]. Ultrasonic elastography is an imaging diagnostic method that deforms a tissue by an external force and estimates elasticity from the strain. However, there is also a problem with the examiner 's dependency and reproducibility. On the other hand, ARFI (Acoustic Radiation Force Impulse) elastography has recently been developed and reported to be more useful and reliable than conventional muscle hardness meter and ultrasonic elastography [24-25]. In ARFI elastography, it is not necessary to move the probe at all to exploit the acoustic effects, and it is less dependent on the examiner's procedure. ARFI can also vibrate deep tissue to generate shear waves and measuring the speed of this shear wave enables quantitative measurement of stiffness that was previously difficult. In fact, Ceyhan Bilgici M, et al. used ARFI elastography to evaluate muscle spasticity after BTA injection in children with Cerebral palsy [25]. Thus, ARFI is a highly reliable and reproducible measurement method, however, that is precision mechanical equipment and the movement of the equipment is not easy. On the other hand, TMG is easy to move, and can be evaluated in various facilities. In the future, it may be necessary to compare the usefulness of ARFI and TMG for the patients after stroke.
This study has some limitations, first of all is the smaller number of cases. Although this study was retrospective observational study of 10 cases without control, comparison of Dm value of biceps brachii muscle between affected side and unaffected side, changes in the Dm value and the VAS value of biceps brachii muscle after BTA administration were similar in all cases. Therefore, even if the number of cases would be increased, the results were considered to be similar. Second, with TMG, only superficial muscles can be measured, and it is difficult to measure the characteristics of muscle contraction of the deep muscles. Although biceps brachii muscle was measured in this study, the above-mentioned ARFI elastography might be more useful for evaluation of deep muscles such as the posterior tibial muscle. Thirdly, as the patient background, diagnosis of the patient and the period from onset to measurement was various, and patients with primary injection and continuous injection were mixed. In addition, rehabilitation after BTA treatment was also varied. In the future, if the number of cases increases, it may be possible to compare the difference between patients with primary injections and with continuous injections and among the type of post-administration rehabilitation.

Although BTA is a very valuable drug, there is no quantitative evaluation assessment for the effects established to date. However, in this study, it was possible to measure the muscle stiffness of the patients after stroke using TMG and to quantitatively evaluate the change of muscle stiffness after administration of BTA. In the future, it is necessary to increase the number of cases and to evaluate the effects after BTA in detail. As a result, it might be possible to establish the guidelines on the dose, site and muscle of administration and interval of administration of BTA, and the clinical significance for BTA treatment is very large.

\section{Conclusion}

For the first time, TMG was used to measure the characteristics of muscle contraction for the patients after stroke quantitatively and to observe the changes of the characteristics after BTA administration. Measurement of the characteristics of muscle contraction using TMG might be useful as one of the quantitative outcome measures for the effect of BTA therapy in patients after stroke.

\section{Conflicts of interest}

There are no conflicts of interest.

\section{References}

1. Sakaguchi G (1984) Structure and function of botulinum toxins. In: Bacterial protein toxins (Alouf JE, et al. eds), Academic Press, London.

2. Scott AB (1981) Botulinum toxin injection of eye muscles to correct strabismus. Trans Am Ophthalmol Soc 79: 734-770. [Crossref]

3. Langdon K, Blair E, Davidson SA, Valentine J (2010) Adverse events following botulinum toxin type A treatment in children with cerebral palsy. Dev Med Child Neurol 52: 972-973. [Crossref]

4. Kerr Graham H, Selber P (2003) Musculoskeletal aspects of cerebral palsy. $J$ Bone Joint Surg Br 85: 157-166. [Crossref]

5. Cosgrove AP, Corry IS, Graham HK (1994) Botulinum toxin in the management of the lower limb in cerebral palsy. Dev Med Child Neurol 36: 386-396. [Crossref]

6. de Paiva A, Meunier FA, Molgo J, Aoki KR, Dolly JO (1999) Functional repair of motor endplates after botulinum neurotoxin type A poisoning: biphasic switch of synaptic activity between nerve sprouts and their parent terminals. Proc Natl Acad Sci US A 96: 3200-3205. [Crossref]

7. Yam WK, Leung MS (2006) Interrater reliability of Modified Ashworth Scale and Modified Tardieu Scale in children with spastic cerebral palsy. J Child Neurol 21: 10311035. [Crossref] 
8. Numanoglu A, Gunel MK (2012) Intraobserver reliability of modified Ashworth scale and modified Tardieu scale in the assessment of spasticity in children with cerebral palsy. Acta Orthop Traumatol Turc 46: 196-200. [Crossref]

9. Mutlu A, Livanelioglu A, Gunel MK (2008) Reliability of Ashworth and Modified Ashworth scales in children with spastic cerebral palsy. BMC Musculoskelet Disord 9: 44. [Crossref]

10. Pereira LA, Ramirez-Campillo R, Martin-Rodriguez S, Kobal R, Abad CCC, et al. (2019) Is Tensiomyography-Derived Velocity of Contraction a Sensitive Marker to Detect Acute Performance Changes in Elite Team-Sport Athletes? Int J Sports Physiol Perform 8: 1-25. [Crossref]

11. Loturco I, Kobal R, Kitamura K, Fernandes V, Moura N, et al. (2019) Predictive Factors of Elite Sprint Performance: Influences of Muscle Mechanical Properties and Functional Parameters. J Strength Cond Res 33: 974-986. [Crossref]

12. Sanchez-Sanchez J, Garcia-Unanue J, Hernando E, Lopez-Fernandez J, Colino E, et al. (2019) Repeated Sprint Ability and Muscular Responses According to the Age Category in Elite Youth Soccer Players. Front Physiol 10: 175. [Crossref]

13. Martin-Rodriguez S, Loturco I, Hunter AM, Rodriguez-Ruiz D, Munguia-Izquierdo D (2017) Reliability and Measurement Error of Tensiomyography to Assess Mechanical Muscle Function: A Systematic Review. J Strength Cond Res 31: 3524-3536. [Crossref]

14. Simunic B (2012) Between-day reliability of a method for non-invasive estimation of muscle composition. J Electromyogr Kinesiol 22: 527-530. [Crossref]

15. Tous-Fajardo J, Moras G, Rodriguez-Jimenez S, Usach R, Doutres DM, et al. (2010) Inter-rater reliability of muscle contractile property measurements using non-invasive tensiomyography. J Electromyogr Kinesiol 20: 761-766. [Crossref]

16. Rey E, Lago-Penas C, Lago-Ballesteros J (2012) Tensiomyography of selected lowerlimb muscles in professional soccer players. J Electromyogr Kinesiol 22: 866-872. [Crossref]
17. Maeda N, Urabe Y, Tsutsumi S, Fujishita H, Numano S, et al. (2018) Symmetry tensiomyographic neuromuscular response after chronic anterior cruciate ligament (ACL) reconstruction. Knee Surg Sports Traumatol Arthrosc 26: 411-417. [Crossref]

18. Alentorn-Geli E, Alvarez-Diaz P, Ramon S, Marin M, Steinbacher G, et al. (2015) Assessment of neuromuscular risk factors for anterior cruciate ligament injury through tensiomyography in male soccer players. Knee Surg Sports Traumatol Arthrosc 23: 2508-2513. [Crossref]

19. Rusu LD, Cosma GG, Cernaianu SM, Marin MN, Rusu PF, et al. (2013) Tensiomyography method used for neuromuscular assessment of muscle training. $J$ Neuroeng Rehabil 10: 67. [Crossref]

20. de Paula Simola RÃ, Harms N, Raeder C, Kellmann M, Meyer T, et al. (2015) Assessment of neuromuscular function after different strength training protocols using tensiomyography. J Strength Cond Res 29: 1339-1348. [Crossref]

21. Ceyhan Bilgici M, Bekci T, Ulus Y, Bilgici A, Tomak L, et al. (2018) Quantitative assessment of muscle stiffness with acoustic radiation force impulse elastography after botulinum toxin A injection in children with cerebral palsy. J Med Ultrason 45: 137141. [Crossref]

22. Ashina M, Bendtsen L, Jensen R, Sakai F, Olesen J (1998) Measurement of muscle hardness: a methodological study. Cephalalgia 18: 106-111. [Crossref]

23. Mathevon L, Michel F, Decavel P, Fernandez B, Parratte B, et al. (2015) Muscle structure and stiffness assessment after botulinum toxin type A injection. A systematic review. Ann Phys Rehabil Med 58: 343-350. [Crossref]

24. Akagi R, Kusama S (2015) Comparison Between Neck and Shoulder Stiffness Determined by Shear Wave Ultrasound Elastography and a Muscle Hardness Meter. Ultrasound Med Biol 41: 2266-2271. [Crossref]

25. Yamamoto A, Yamakoshi Y, Ohsawa T, Shitara H, Ichinose T, et al. (2018) Shear wave velocity measurement of upper trapezius muscle by color Doppler shear wave imaging. J Med Ultrason 45: 129-136. [Crossref]

Copyright: (92019 Mikami Y. This is an open-access article distributed under the terms of the Creative Commons Attribution License, which permits unrestricted use, distribution, and reproduction in any medium, provided the original author and source are credited. 